\title{
A new layered MIMO-OFDM system with Signal Space Diversity
}

\author{
Jin-Yong Choi ${ }^{1 \mathrm{a})}$, Jong-Seob Baek ${ }^{2}$, and Jong-Soo Seo ${ }^{1}$ \\ ${ }^{1}$ Department of Electrical and Electronic Engineering, \\ Yonsei University, Seoul 120-749, South Korea \\ ${ }^{2}$ Department of Electrical and Computer Engineering, \\ Georgia Institute of Technology, Atlanta, GA, 30309, USA \\ a) jeeney@yonsei.ac.kr
}

\begin{abstract}
This paper aims to design a new transceiver with a signal space diversity (SSD) in layered multiple-input multiple-output (MIMO) orthogonal frequency division multiplexing (OFDM) systems. To this end, a enhanced coordinate interleaver (E-CI) that swaps a random pair of subcarriers is first proposed in order to realize readily the detection ordering required for a layered MIMO processing at the receiver. A enhanced maximum likelihood (E-ML) detection is also proposed in order to provide SSD gain after the MIMO detection processing. The performance of layered MIMO-OFDM system is evaluated and compared with that of MIMO-OFDM systems without the E-CI and E-ML detection over a time-varying Typical Urban (TU) channel.
\end{abstract} Keywords: coordinate interleaver, maximum likelihood detection, MIMO-OFDM, signal space diversity

Classification: Wireless circuits and devices

\section{References}

[1] H. Bolcskei and E. Zurich, "MIMO-OFDM Wireless System: BASICS, Perspectives and Challenges," IEEE Wireless Commun., vol. 13, no. 4, pp. 31-37, Aug. 2006.

[2] J. Boutros and E. Viterbo, "Signal space diversity: a power and bandwidth-efficient technique for the Rayleigh fading channel," IEEE Trans. Inf. Theory, vol. 44, no. 4, pp. 1453-1467, July 1998.

[3] M. N. Khormuji, U. H. Rizvi, G. J. M. Janssen, and S. B. Slimane, "Rotation optimization for MPSK/MQAM signal constellations over Rayleigh fading channels," Proc. IEEE ICCS, pp. 1-5, 2006.

[4] J. Kim and I. Lee, "Coded layered space-time transmission with signal space diversity in OFDM systems," Proc. IEEE GLOBECOM, pp. 38663870, Dec. 2005.

[5] S. J. Hong, J. Y. Choi, S. U. Hwang, S. W. Jeon, and J. S. Seo, "Interleaved spatial diversity transmission with coordinate interleaver for MIMO-OFDM systems," Proc. IEEE VTC, pp. 1-4, April 2009.

[6] P. W. Wolniansky, G. J. Foschini, G. D. Golden, and R. A. Valenzuela, "V-BLAST: an architecture for realizing very high data rates over the richscattering wireless channel," URSI International Symposium on Signals, Systems and Electronics, pp. 295-300, Sept. 1998. 
[7] COST-207 Final Report, "Digital land mobile radio communications," COST 207 Management Committee, 1998.

[8] R. U. Nabar, H. Bolcskei, and A. J. Paulraj, "Diversity and outage performance in space-time block coded Ricean MIMO channels," IEEE Trans. Wireless Commun., vol. 4, no. 5, pp. 2519-2532, Sept. 2005.

\section{Introduction}

A layered multiple-input multiple-output (MIMO) orthogonal frequency division multiplexing (OFDM) and a signal space diversity (SSD) have been regarded as the promising techniques to provide high-data rate and highquality services in fading channels $[1,2,3,4]$. In particular, it was shown in $[2,3]$ that the SSD provides a diversity gain without a loss of the spectral efficiency even though the signal experiences a deep null fading in a singleinput single-output (SISO) system. The achievement is actually realized by exploiting a rotated constellation $(\mathrm{RC})$ that rotates the real and imaginary components of a constellation point, a coordinate interleaver (CI) that swaps the position of a component randomly at transmitter, and a maximum likelihood (ML) detection that only exploits a magnitude information of a channel at the receiver.

Recently, it was shown in [4] that a diversity gain can be also obtained by applying such RC and CI schemes to the layered MIMO-OFDM system equipped with an ordered minimum mean squared error (MMSE) detection. Unfortunately, the diversity gain is a partial SSD gain induced by the RC and CI, and there is no diversity gain with MMSE detection $[2,3]$. In addition, it is found that the detection ordering for a layered MIMO processing is limited to a special approach for improving channel capacity. That is, the use of the well reviewed detection ordering exploiting a channel state information (CSI) to such a system is not easy because of the CSI dispersion induced by the CI. Furthermore, we assert that these problems could be encountered when the SSD is combined with a MIMO system.

In order to resolve the aforementioned limitations, this paper presents a new layered MIMO-OFDM system with SSD by proposing a enhanced CI (E-CI) and a enhanced ML (E-ML) detection. The E-CI swaps a random pair of subcarriers so as to make a detection ordering exploit the CSI. The E-ML detection provides SSD gain by compensating a magnitude effect of the CSI that is canceled by the layered MIMO processing. The performance evaluation is then accomplished in a time-varying Typical Urban (TU) MIMO channel.

\section{Proposed MIMO-OFDM transmitter}

We present the proposed MIMO-OFDM transmitter with SSD that consists of a symbol mapper, RC, E-CI, and $N_{t}$ layer transmission blocks as depicted in Fig. 1. The output symbol vector $\widehat{\mathbf{s}}_{i}$ of a symbol mapper on the $i$ th layer 


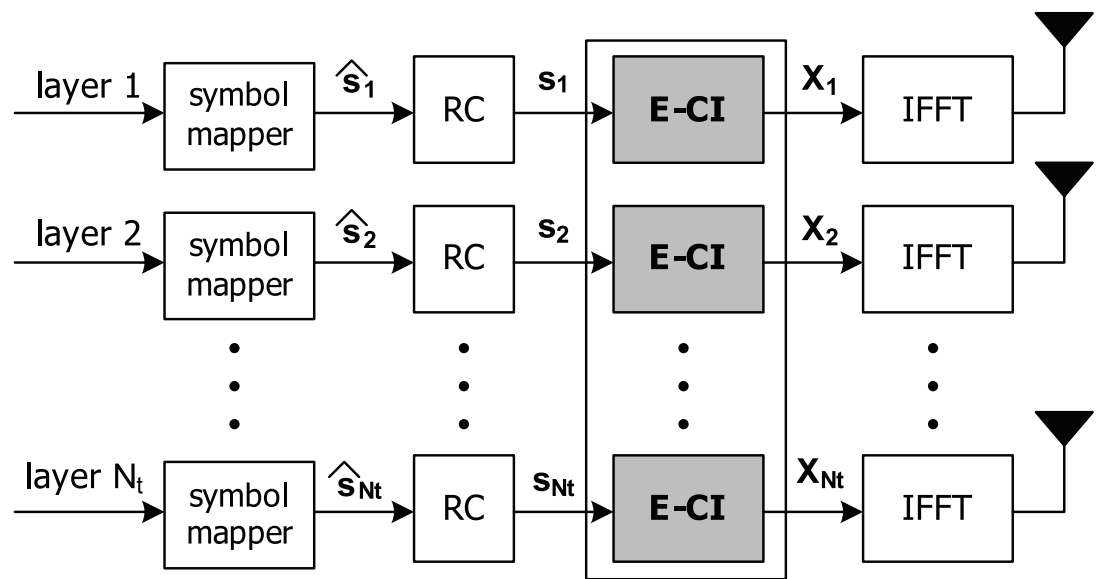

$\mathrm{RC}$ : rotated constellation

E-CI: enhanced coordinated interleaver

\begin{tabular}{|c|c|c|c|c|c|c|}
\hline $\mathrm{s}_{\mathrm{i}, 1}{ }^{\mathrm{R}}$ & $\bullet \bullet \bullet$ & $\mathrm{s}_{\mathrm{i}, \mathrm{a}}{ }^{\mathrm{R}}$ & $\bullet \bullet \bullet$ & $\mathrm{s}_{\mathrm{i}, \mathrm{b}}{ }^{\mathrm{R}}$ & $\bullet \bullet \bullet$ & $\mathrm{s}_{\mathrm{i}, \mathrm{Nc}}{ }^{\mathrm{R}}$ \\
\hline $\mathrm{s}_{\mathrm{i}, 1}{ }^{\mathrm{I}}$ & $\bullet \bullet \bullet$ & $\mathrm{s}_{\mathrm{i}, \mathrm{a}}{ }^{\mathrm{I}}$ & $\bullet \bullet \bullet$ & $\mathrm{s}_{\mathrm{i}, \mathrm{b}}{ }^{\mathrm{I}}$ & $\bullet \bullet \bullet$ & $\mathrm{s}_{\mathrm{i}, \mathrm{Nc}} \mathrm{I}^{\mathrm{V}}$ \\
\hline
\end{tabular}

(a)

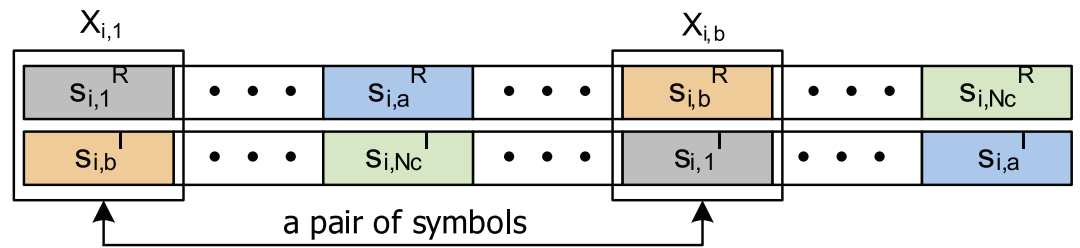

(b)

Fig. 1. The proposed MIMO-OFDM transmitter with SSD; (a) the input symbol vector of E-CI, (b) the output symbol vector of E-CI.

(for $\left.i=1, \ldots, N_{t}\right)$ is defined as

$$
\widehat{\mathbf{s}}_{i}=\left[\begin{array}{lll}
\widehat{\mathrm{s}}_{i, 1} \widehat{\mathrm{s}}_{i, 2} & \cdots & \widehat{\mathrm{s}}_{i, N_{c}}
\end{array}\right]^{T}
$$

where the superscript $T$ denotes a vector transpose operation, and $N_{c}$ denotes the length of an inverse fast Fourier transform (IFFT) and E-CI [ref. (3)]. The RC block rotates the real and imaginary components, i.e., $\widehat{\mathrm{s}}_{i, p}^{R}$ and $\widehat{\mathrm{s}}_{i, p}^{I}$, of the $p$ th symbol $\widehat{\mathrm{s}}_{i, p}$ with an angle $\theta(0 \leq \theta<\pi / 2)$ as follows

$$
\left[\begin{array}{c}
\mathrm{s}_{i, p}^{R} \\
\mathrm{~s}_{i, p}^{I}
\end{array}\right]=\left[\begin{array}{rr}
\cos \theta & -\sin \theta \\
\sin \theta & \cos \theta
\end{array}\right]\left[\begin{array}{c}
\widehat{\mathrm{s}}_{i, p}^{R} \\
\widehat{\mathrm{s}}_{i, p}^{I}
\end{array}\right]
$$

where the superscripts $R$ and $I$ denote the real and imaginary components, respectively. Then, the symbol vector $\mathbf{s}_{i}=\left[\begin{array}{llll}\mathrm{s}_{i, 1} & \mathrm{~s}_{i, 2} & \cdots & \mathrm{s}_{i, N_{c}}\end{array}\right]$, where $\mathrm{s}_{i, k}=$ $\mathrm{s}_{i, k}^{R}+j \mathrm{~s}_{i, k}^{I}$, passes through the E-CI as shown in Fig. 1(a)-(b). Let us define the output symbol vector of E-CI as $\mathbf{X}_{i}=\left[\begin{array}{llll}\mathrm{X}_{i, 1} & \mathrm{X}_{i, 2} & \cdots \mathrm{X}_{i, N_{c}}\end{array}\right]^{T}$. The $p$ th interleaved subcarriers $\mathrm{X}_{i, p}$ that corresponds to the $p$ th subcarrier of an OFDM symbol is given by

$$
\mathrm{X}_{i, p}=\mathrm{s}_{i, p}^{R}+j \mathrm{~s}_{i, q}^{I}, \text { for } p, q=1, \ldots, N_{c}, \text { and } p \neq q .
$$


For the comprehensive understanding of E-CI, we illustrate the swapping operation in Fig. 1(a)-(b). It is shown that the E-CI chooses randomly a pair of subcarriers, i.e., the 1st and the bth subcarriers, and then swaps only the imaginary component as follows

$$
\mathrm{X}_{i, 1}=\mathrm{s}_{i, 1}^{R}+j \mathrm{~s}_{i, b}^{I}, \text { and } \mathrm{X}_{i, b}=\mathrm{s}_{i, b}^{R}+j \mathrm{~s}_{i, 1}^{I} .
$$

The E-CI can be differentiated from a conventional CI (C-CI) in which all of imaginary components are interleaved randomly on the each layer. In Fig. 1, after passing IFFT block and inserting a guard-interval (GI) of length $N_{g}$, an OFDM symbol is transmitted.

\section{Proposed MIMO-OFDM receiver}

We develop the receiver processing on the $p$ th subcarriers of all of the antennas [4]. After removing the GI and passing through FFT block, the receive symbol vector is given by

$$
\mathbf{Y}_{p}=\mathbf{H}_{p} \mathbf{X}_{p}+\mathbf{N}_{p}
$$

where $\mathbf{X}_{p}=\left[\begin{array}{lll}\mathrm{X}_{1, p} & \mathrm{X}_{2, p} \cdots \mathrm{X}_{N_{t}, p}\end{array}\right]^{T}, \mathbf{Y}_{p}=\left[\begin{array}{lll}\mathrm{Y}_{1, p} & \mathrm{Y}_{2, p} \cdots \mathrm{Y}_{N_{r}, p}\end{array}\right]^{T}$, and $\mathbf{N}_{p}=$ $\left[\mathrm{N}_{1, p} \mathrm{~N}_{2, p} \cdots \mathrm{N}_{N_{r}, p}\right]^{T}$ denotes an additive white Gaussian noise (AWGN) vector. $N_{r}$ is the number of antenna in the MIMO-OFDM receiver. The channel frequency response (CFR), $\mathbf{H}_{p}$, is defined as a matrix of size $N_{r} \times N_{t}$ as follows

$$
\mathbf{H}_{p}=\left(\begin{array}{ccc}
\mathrm{H}_{1,1, p} & \ldots & \mathrm{H}_{1, N_{t}, p} \\
\mathrm{H}_{2,1, p} & \ldots & \mathrm{H}_{2, N_{t}, p} \\
\vdots & \ddots & \vdots \\
\mathrm{H}_{N_{r}, 1, p} & \ldots & \mathrm{H}_{N_{r}, N_{t}, p}
\end{array}\right)
$$

where $\mathrm{H}_{k, i, p}$ denotes the $p$ th CFR component between the $i$ th transmit antenna and the $k$ th receive antenna.

Fig. 2 depicts the proposed receiver processing. In the layered MIMO detection processing, the detection ordering is first determined and then the $p$ th receive subcarriers are layered by using a successive interference cancellation (SIC) and a zero-forcing (ZF) detection ${ }^{1}$. Finally, the proposed E-ML detection is performed for each layered subcarrier after performing a deinterleaving that corresponds to the E-CI. That is, the information related to $\mathrm{E}-\mathrm{CI}$ in deinterleaving process is known.

\subsection{Proposed detection ordering}

The detection ordering is usually needed to improve the system performance by detecting a reliable subcarrier with a priority $[4,5,6]$. To this end, we exploit the basic principle of well-known detection ordering of [6] in which the order is determined based on a channel inverse matrix for use in the ZF detection. That is, by defining that $\left(\mathbf{H}_{p}\right)^{-1}=\mathbf{G}_{p}$ and the $i$ th row vector of

\footnotetext{
${ }^{1}$ Though the ZF detection is applied in this paper, the MMSE detection can be also used.
} 


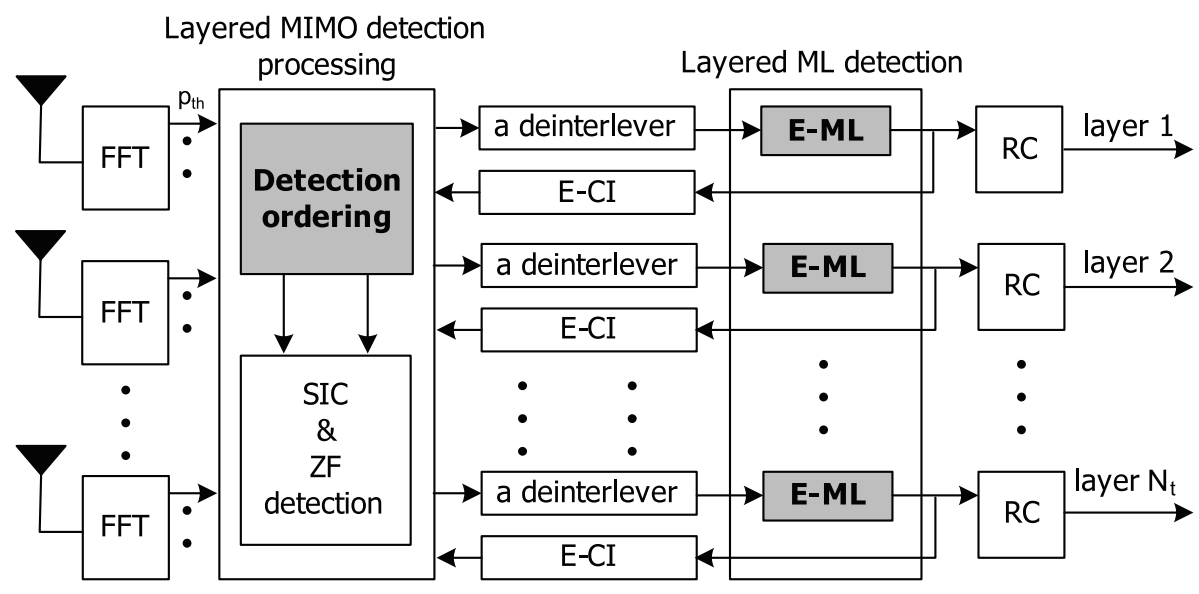

Fig. 2. The proposed MIMO-OFDM receiver.

$\mathbf{G}_{p}$ is $\mathbf{w}_{p, i}=\left[\mathbf{G}_{p}\right]_{i}$, a subcarrier of the $i$ th layer having the smallest norm value of $\left\|\mathbf{w}_{p, i}\right\|^{2}$ takes the highest priority in the detection ordering. Then, the subcarrier is detected and its effect to the other layers is canceled. Such a procedure is repeated until all of the subcarriers are detected as the layered MIMO detection processing shown in Fig. 2. Unfortunately, the detection ordering would not be suitable for use in MIMO-OFDM system with SSD due to the fact that the reliability of $\left\|\mathbf{w}_{p, i}\right\|^{2}$ cannot be guaranteed after passing the deinterleaver. Hence, the reliability of detection ordering needs to be improved by considering the effect of interleaver. From Fig. 1 (a)-(b), the detection ordering can be performed effectively by adding $\left\|\mathbf{w}_{q, i}\right\|^{2}$ and $\left\|\mathbf{w}_{p, i}\right\|^{2}$ as follows

$$
\mathbf{O}(m)=\min _{i}\left(\left\|\mathbf{w}_{p, i}\right\|^{2}+\left\|\mathbf{w}_{q, i}\right\|^{2}\right) \text { for } i=1, \ldots, N_{r}
$$

where $\mathbf{O}(m)$ denotes the layer index with the $m$ th detection order and it is decided by comparing the sum of norms of each layer. Here, we assert from (7) that the detection ordering is readily realized by the E-CI.

\subsection{Proposed E-ML detection}

We present the E-ML detection to provide SSD gain by compensating the magnitude information of the CSI, which is canceled by the layered MIMO detection processing ${ }^{2}$. It is known that SSD gain of E-ML detection is generated by using only a magnitude information (not phase information) of channel $[2,3]$. In the followings, it is assumed that the output symbol vector of the $i$ th deinterleaver is $\widehat{\mathbf{y}}_{i}=\left[\widehat{\mathrm{y}}_{i, 1} \widehat{\mathrm{y}}_{i, 2} \cdots \widehat{\mathrm{y}}_{i, N_{c}}\right]^{T}$ where $\widehat{\mathrm{y}}_{i, p}=\widehat{\mathrm{y}}_{i, p}^{R}+j \widehat{\mathrm{y}}_{i, p}^{I}$. It is also assumed that the components $\widehat{\mathrm{y}}_{i, p}^{R}$ and $\widehat{\mathrm{y}}_{i, p}^{I}$ experience CFRs $\mathrm{H}_{k, i, p}$ and $\mathrm{H}_{k, i, q}$ in (6), respectively. Correspondingly, the E-ML detection on $\widetilde{\mathrm{s}}_{i, p}$ is defined as

$$
\widetilde{\mathrm{s}}_{i, p}=\arg \min _{\mathrm{s}_{i, p}}\left(|| \mathrm{H}_{k, i, p}\left|\widehat{\mathrm{y}}_{i, p}^{R}-\right| \mathrm{H}_{k, i, p}\left|\mathrm{~s}_{i, p}^{R}\right|^{2}+|| \mathrm{H}_{k, i, q}\left|\widehat{\mathrm{y}}_{i, p}^{I}-\right| \mathrm{H}_{k, i, q}\left|\mathrm{~s}_{i, p}^{I}\right|^{2}\right)
$$

where $\widetilde{\mathrm{s}}_{i, p}$ is the estimated symbol and the minimization is performed over all possible rotated constellation points on each layer.

\footnotetext{
${ }^{2}$ In this paper, it is noticed that the layered MIMO detection processing is regarded as a pre-processing to layer each subcarrier for use in the E-ML detection, which is different from conventional MIMO-OFDM systems of $[1,4,6]$.
} 


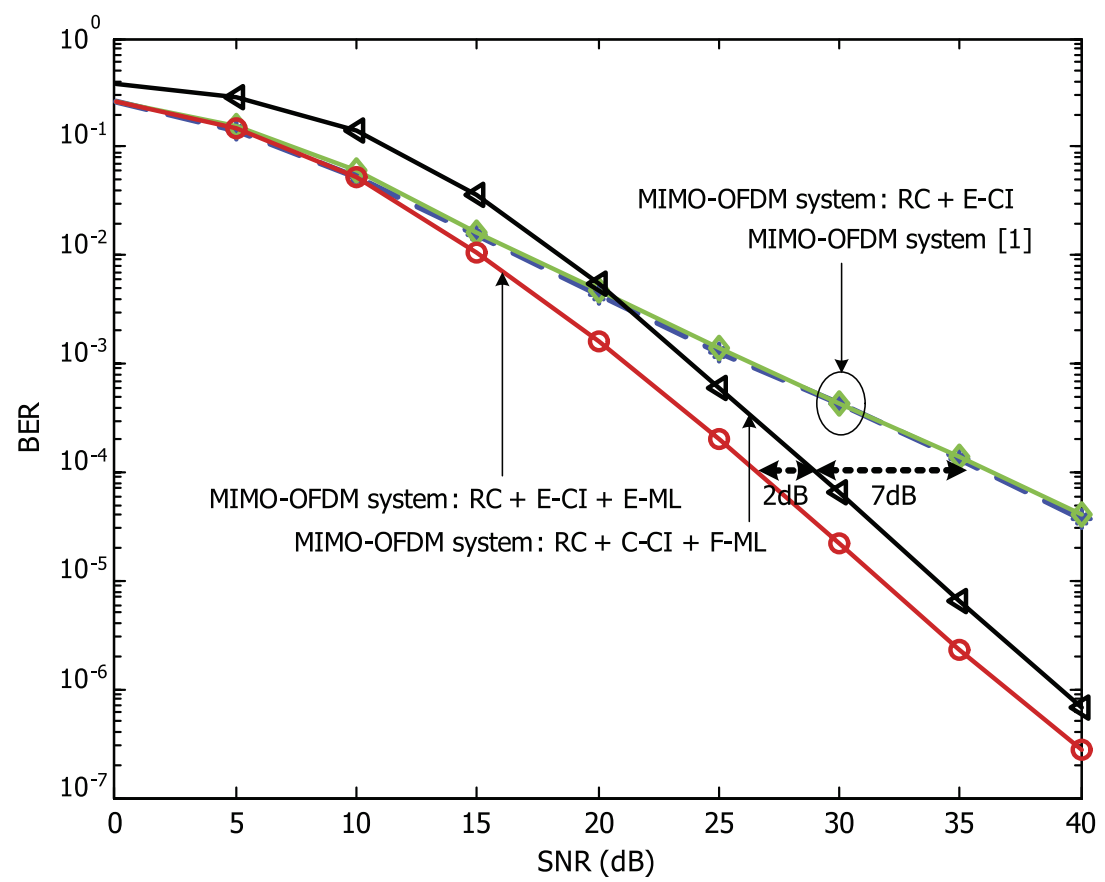

Fig. 3. BER performance of layered MIMO-OFDM systems.

\section{Performance evaluations}

The performance evaluations are accomplished to analyze the SSD gain of the proposed E-CI and E-ML detection in layered MIMO-OFDM systems over COST 207 typical urban (TU) channel which models a metropolitan area with relatively strong multipaths [7]. For the simulation, it was assumed that the symbol is mapped by QPSK signaling, $N_{c}=256, N_{g}=32, N_{t}=N_{r}=2$, and $\theta=\pi / 6[5]$, a signal bandwidth is $2.56 \mathrm{MHz}$, and Doppler frequency $f_{d}=20 \mathrm{~Hz}$. It was also assumed that a perfect CSI is known to the receiver.

Fig. 3 shows a bit-error ratio (BER) performance of various layered MIMO-OFDM systems: 1) MIMO-OFDM system [1], 2) MIMO-OFDM system employing the RC and E-CI, 3) MIMO-OFDM system employing the RC, E-CI and E-ML. It was assumed for all of systems that the detection ordering is based on $\left[\mathbf{G}_{p}\right]_{i}$ and MIMO processing is based on the ZF detection. In addition, we consider a MIMO-OFDM system employing the $\mathrm{RC}, \mathrm{C}-\mathrm{CI}$ and full ML (F-ML), where MIMO detection, instead of the F-ML detects a symbol minimizing an Euclidean distance over all possible rotated constellation points of all the layers. From Fig. 3, it is found that the MIMO-OFDM system and MIMO-OFDM system employing the RC and E-CI have a similar BER performance. However, this result verifies that the E-CI makes it possible for the detection ordering to exploit the $\mathrm{CSI}^{3}$. On the other hand, it is observed that the MIMO-OFDM system employing the RC, E-CI and E-ML outperforms the other two systems by up to SNR of $7 \mathrm{~dB}$ at BER $=10^{-4}$. Moreover, the slope of it's BER curve is getting steeper as SNR increases as

\footnotetext{
${ }^{3}$ The performance of MIMO-OFDM system employing the RC and C-CI is not shown in Fig. 3. However, there should be a severe performance degradation due to the detection ordering.
} 
compared to the MIMO-OFDM system employing the RC and E-CI because of the SSD gain of the E-ML detection [8]. It is also observed that it has even better BER performance than MIMO-OFDM system employing the RC, C$\mathrm{CI}$ and F-ML. Here, the performance degradation of MIMO-OFDM system employing the RC, C-CI and F-ML comes from the fact that it seems like the modulation order of the symbol after passing C-CI is increased because of the affect of $\mathrm{RC}$ and $\mathrm{C}-\mathrm{CI}$.

\section{Conclusions}

This paper has presented enhanced coordinate interleaver (E-CI) and enhanced maximum likelihood (E-ML) detection for use in a layered MIMOOFDM system with SSD. In particular, the E-CI detection was proposed to realize readily the detection ordering in the detection processing of MIMO system. E-ML was proposed to obtain SSD gain in the MIMO-OFDM system. The performance evaluations have verified that the E-CI and E-ML detection could improve the BER performance of layered MIMO-OFDM system with SSD.

\section{Acknowledgments}

This work was supported by the IT R\&D program of MKE/KEIT [KI002091, Research on Multiple Antenna and Multi-hop Relay Transmission Technologies for Next Generation Mobile Broadcasting Service]. 